\title{
Exploring the dimensions of metapopulation persistence : a comparison of structural and temporal measures
}

\section{Dallas, Tad A.}

2021-06

Dallas , T A , Saastamoinen , M \& Ovaskainen , O 2021, ' Exploring the dimensions of metapopulation persistence : a comparison of structural and temporal measures ' , Theoretical ecology. , vol. 14 , pp. 269-278 . https://doi.org/10.1007/s12080-020-00497-0

http://hdl.handle.net/10138/338361

https://doi.org/10.1007/s12080-020-00497-0

other

acceptedVersion

Downloaded from Helda, University of Helsinki institutional repository.

This is an electronic reprint of the original article.

This reprint may differ from the original in pagination and typographic detail.

Please cite the original version. 


\title{
Exploring the dimensions of metapopulation persistence: a comparison of structural and temporal measures
}

\author{
Tad A Dallas ${ }^{\mathrm{a}, \mathrm{b},},{ }^{*}$, Marjo Saastamoinen ${ }^{\mathrm{b}, \mathrm{c}}$ and Otso Ovaskainen ${ }^{\mathrm{b}, \mathrm{d}}$ \\ a Department of Biological Science, Louisiana State University, Baton Rouge, LA, USA \\ ${ }^{b}$ Organismal and Evolutionary Biology Research Programme, P.O. Box 65, 00014 University of Helsinki, Finland \\ ${ }^{\mathrm{c}}$ Helsinki Institute of Life Science, University of Helsinki, Finland \\ ${ }^{\mathrm{d} C e n t r e ~ f o r ~ B i o d i v e r s i t y ~ D y n a m i c s, ~ D e p a r t m e n t ~ o f ~ B i o l o g y, ~ N o r w e g i a n ~ U n i v e r s i t y ~ o f ~ S c i e n c e ~ a n d ~ T e c h n o l o g y . ~}$ \\ N-7491 Trondheim, Norway
}

*Corresponding author: tad.a.dallas@gmail.com; ORCID: 0000-0003-3328-9958 


\section{Author Declarations}

Ethics approval and consent to participate: This work required no ethics approval, and all authors contributed to this project.

Consent for publication: All authors approved the submission of this work.

Availability of data and materials: $R$ code is available on figshare at https://doi.org/10.6084/m9.figshare.12576038.

Competing interests: The authors have no conflicts of interest to declare.

Funding: The research was funded by the Academy of Finland (grant 309581 to OO), the Research Council of Norway (SFF-III grant 223257), and the European Research Council (Independent Starting grant no. 637412 'META-STRESS' to MS)

Authors' contributions: TAD designed the study and performed the analyses. All authors contributed to manuscript writing.

Acknowledgements: We thank the coordinators and volunteers who participated in the Åland island survey since 1993. The research was funded by the Academy of Finland (grant 309581 to OO), the Research Council of Norway (SFF-III grant 223257), and the European Research Council (Independent Starting grant no. 637412 'META-STRESS' to MS). The Research Centre for Ecological Change is funded by the Jane and Aatos Erkko Foundation. TAD thanks the Department of Mathematics at University of Rijeka for their hospitality.

Authors' information: Tad Dallas; tad.a.dallas@gmail.com; ORCID: 0000-0003-3328-9958

Running title: Metapopulations and power laws

Keywords: Metapopulation, Spatial network, Persistence, Power law, Extinction time 
The spatial arrangement of habitat patches in a metapopulation, and the dispersal connections among them, influence metapopulation persistence. Metapopulation persistence emerges from a dynamic process, namely the serial extinctions and recolonizations of local habitat patches, while measures of persistence are typically based solely on structural properties of the spatial network (e.g., spatial distance between sites). Persistence estimators based on static properties may be unable to capture the dynamic nature of persistence. Understanding the shape of the distribution of extinction times is a central goal in population ecology. Here, we examine the goodness of fit of the power law to patch persistence time distributions using data on a foundational metapopulation system - the Glanville fritillary butterfly in the Aland islands. Further, we address the relationship between structural measures of metapopulation persistence (i.e., metapopulation capacity) and our temporal distributional fits to patch persistence times based on a power law. Patch persistence time distributions were well fit by a power law for the majority of semi-independent networks. Power law fits to persistence time distributions were related to metapopulation capacity, linking structural and temporal measures of metapopulation persistence. Several environmental variables and measures of network topology were correlated with both measures of metapopulation persistence, though correlations tended to be stronger for the structural measure of metapopulation persistence (i.e., metapopulation capacity). Together, our findings suggest that persistence time distributions are useful dynamic properties of metapopulations, and provide evidence of a relationship between metapopulation structure and metapopulation dynamics. 
Habitat fragmentation reduces patch size leading to smaller local populations that are at greater risk of stochastic extinction. Dispersal between fragmented suitable habitat patches is therefore essential to maintain the network of small populations i.e., the metapopulation (Hanski, 1999; Hanski and Gilpin, 1991). A body of theory has been developed to describe threshold conditions for metapopulation persistence (Keymer et al., 2000), the influence of dispersal on metapopulation dynamics (Doebeli, 1995; Holland and Hastings, 2008; Vuilleumier et al., 2010), and the dependence of persistence on the spatial distribution of habitat patches (Ovaskainen and Hanski, 2003). Metapopulation persistence estimators attempt to quantify the threshold after which colonization and dispersal are unable to maintain patch occupancy, leading to metapopulation collapse. The initial development of metapopulation theory drew heavily on the Levin's model, which tracks species occurrences among patches regardless of spatial location or size of habitat patches (Levins, 1969). Building on this, Hanski (1994) developed a spatially-explicit metapopulation model which incorporated variation in habitat patch size and explicitly considered the role of space. Through this work, the development of a persistence measure called metapopulation capacity was developed (Hanski and Ovaskainen, 2000; Ovaskainen and Hanski, 2001).

- We use metapopulation capacity to measure metapopulation persistence based on the spatial distribution of habitat patches and dispersal links between them (Hanski and Ovaskainen, 2000; Ovaskainen and Hanski, 2001). This information is contained within the landscape matrix (M), which describes the putative dispersal links between all habitat patches (Hanski, 1999; Ovaskainen and Hanski, 2001). While the landscape matrix is often constructed in the absence of a dynamic model, the original formulation of metapopulation capacity $(\lambda)$ was based on a metapopulation model (Hanski and Ovaskainen, 2000). Previous work has suggested that metapopulation capacity $(\lambda)$ is associated with equilibrium patch occupancy when habitat patches are of good quality and are aggregated in space (Hanski and Ovaskainen, 2000; Visconti and Elkin, 2009), suggesting a role for both environmental quality and spatial network structure on metapopulation capacity (Hanski et al., 2017). Conservation and management decisions have 
been informed by metapopulation capacity (Hanski, 2011; Hanski and Thomas, 1994; McCullough, 1996), as it is used to estimate long term metapopulation persistence (Hanski and Ovaskainen, 2000). Similar eigenvalue-decompositions have been used to estimate $a$ ) epidemic thresholds in social contact networks (Saha et al., 2015), b) nestedness in bipartite networks (Staniczenko et al., $2013), c)$ the basic reproductive number $\left(R_{0}\right)$ of infectious disease given infection time series (Diekmann et al., 2010), and d) early warning signals of spatial population collapse (Chen et al., 2019).

- Previous efforts to link metapopulation persistence measures derived from the spatial distribution of patches to the resulting dynamics have largely focused on metapopulation persistence in an absolute sense, quantifying the number of times in model simulations the metapopulation goes extinct (Kleinhans and Jonsson, 2011). Other efforts have defined persistence using measures related to extinction-colonization ratios or mean species occupancy (i.e., fraction of patches where the species is found). These measures often define metapopulation persistence as either the probability that the entire metapopulation goes extinct, or the mean species occupancy over some time window (Johst et al., 2002; Molofsky and Ferdy, 2005). The first is a coarse measure, and is difficult to empirically test, given the need for a metapopulation extinction event, which tend to be rare and not easily replicated. The second measure may fail to capture rescue effects or transient patch occupancy followed by local extinction, that may serve as an early warning signal of metapopulation collapse (but see Holmes et al. (2020)). Ideally, a measure of persistence at the metapopulation scale would incorporate information on each habitat patch explicitly, both in terms of mean and variation in climatic conditions. For instance, Increasing climatic variability may drive metapopulation dynamics near extinction thresholds, even those as established as the Glanville fritillary metapopulation in the Åland islands (van Bergen et al., 2020).

- In population ecology, a body of theory related to the distribution of extinction times for single populations has been developed (Drake, 2006, 2014). That is, without immigration or emigration, what does the distribution of extinction times look like for a set of populations? A common observation is that this distribution has a heavy tail, where most populations go 
extinct in a relatively short time, but few populations exist for far longer (Drake, 2014). In the context of metapopulations, the distributional fit to patch extinction times - which are equivalent to persistence times - may provide information on the metapopulation as a whole while directly incorporating patch level dynamics (Bertuzzo et al., 2011). This approach requires either simulated or empirical data on patch persistence times to generate the persistence estimate. That is, estimating the distribution of persistence times for each patch in the network could provide insight into the presence of long-persisting nodes, and those which go extinct but recolonize quickly. One such distributional fit proposed recently is the power law (Bertuzzo et al., 2011), where some quantity $x$ is drawn from a probability distribution $p(x) \propto x^{-\alpha}$. The interpretation of $\alpha$ then becomes important, as this scaling parameter starts to address the heavy-tailed nature of the distribution of empirical values of $x$. Power law relationships are commonly found in natural systems, such as the bivariate scaling of the number of species with increasing geographic area (the species-area relationship; Martín and Goldenfeld (2006)). Power law relationships in frequency distributions, as examined here, are equally common in ecological studies of the distribution of species body sizes (Morse et al., 1985), abundance estimates (Keitt and Stanley, 1998), and vegetation patch size (Kéfi et al., 2007), as reviewed in White et al. (2008).

. Here, the parameter $\alpha$ estimates the shape of the long tail of persistence times, with smaller $\alpha$ values corresponding to heavier tails. This means that large $\alpha$ values correspond to more extreme decay rates in persistence times $(x)$, with very few long persistent patches, indicative of high extinction and rapid recolonization of habitat patches. That is, the probability density of persistence times $(x)$ are proportional to $x^{-\alpha}$. As such, there are two clear possible relationships between metapopulation capacity $(\lambda)$ and persistence time distributions $(\alpha)$. First, a positive relationship may emerge between persistence time distributional fits $(\alpha)$ and metapopulation capacity $(\lambda)$ if long-term persistent habitat patches drive metapopulation persistence. These long-term persistent patches would lead to a heavier-tailed distribution of persistence times, reducing the $\alpha$ value. On the other hand, metapopulations are characterized by rapid extinction and re-colonization dynamics, and these dynamics may be indicative of a persistent metapopulation. Thus, a second possibility is that we may expect a negative relationship between metapopulation 
capacity $(\lambda)$ and persistence time fits $(\alpha)$. This would suggest that short-lived, but quickly recolonized habitat patches, are a signature of a persistent metapopulation.

Apart from implications to metapopulation persistence, the $\alpha$ parameter may also be useful in differentiating different types of metapopulations (as identified in Harrison and Taylor (1997)). This is because the balance between ephemeral and persistant habitat patches can inform metapopulation structure. For instance, mainland-island metapopulations would be expected to have a smaller $\alpha$ value, driven by the long-persisting source patches, while classic metapopulations would have larger $\alpha$ values due to the common extinction and colonization events reducing the probability of long-persisting patches. Finally, understanding the differences in power law relationships for unconnected populations (Drake, 2006, 2014) - corresponding to non-equilibrium metapopulations as defined in Harrison and Taylor (1997) - and connected metapopulations can provide insight into the role of dispersal and rescue effects on persistence.

. How well do structural (metapopulation capacity) or temporal (persistence time distributions) measures of metapopulation persistence describe metapopulation dynamics, given that they both putatively quantify metapopulation persistence? A common assumption of many metapopulation studies is that structural properties of the metapopulation (e.g., metapopulation capacity) capture dynamic processes (Hanski and Ovaskainen, 2000). That is, a positive relationship between structural (metapopulation capacity) and temporal (persistence time distributional fit) measures of metapopulation persistence should exist. Further, the relationships between environmental and topological aspects of the metapopulation should correlate well with both measures of metapopulation persistence, though perhaps with different strength. We would expect that measures of spatial network topology (e.g., connectance) should strongly correlate with metapopulation capacity $(\lambda)$, as the both measures are based on the same data (i.e., the landscape matrix $\mathbf{M}$ ). However, factors influencing distributional fits to persistence times should correspond more to local environmental conditions and resource availability, as measures of metapopulation persistence that are based on local dynamics are likely to be more sensitive to local environmental conditions than measures based on metapopulation structure alone. 
- Here, we examine the relationship between structural (metapopulation capacity) and temporal (persistence time distributions) measures of metapopulation persistence, providing a link between the geographic distribution of habitat patches and the resulting temporal metapopulation dynamics. Further, we explore power law scaling relationships in patch persistence times, providing evidence that interconnected populations have similarly heavy-tailed persistence (or extinction) time distributions compared to isolated replicated populations (Drake, 2014). Using a long-term sampling effort of Melitaea cinxia populations distributed across meadow habitats in the Åland islands sampled over 20 years, we demonstrate a positive relationship our measures of metapopulation persistence for a set of 88 semi-independent spatial networks (also referred to as network components). Further, we investigate how environmental and topological aspects of the spatial network are related to both measures of metapopulation persistence. Environmental characteristics, such as mean resource availability and grazing pressure, were largely unrelated to either measure of metapopulation persistence, while topological properties - such as modularity and the number of patches in the network - were strongly correlated to both measures of metapopulation persistence. Together, this provides a link between structural and temporal measures of metapopulation persistence, and demonstrates clear relationships between aspects of the landscape matrix and the resulting measures of metapopulation persistence, either measured using putative dispersal connections, or through a power law scaling relationship in patch persistence times.

\section{Methods}

\section{Glanville fritillary metapopulation data}

In the Åland islands, a set of approximately 4500 habitat patches have been monitored since 1993. Here, we use data from the Fall surveys of the Glanville fritillary butterfly (Melitaea cinxia) nests sampled annually between 1993 and 2016 (Ojanen et al., 2013). Each habitat patch was occupied by at least one of two host plant species - either Plantago lanceolata or Veronica spicata - which serves as a food and oviposition resource for M. cinxia. Habitat patches exist in a mosaic of inhospitable habitat, and links between habitat patches represent potential dispersal pathways. 
We examined a subset of 2249 habitat patches which contained sufficient data, leading to the creation of 88 semi-independent networks (SINs). Each SIN has been identified to be a cluster of patches where most of the dispersal dynamics are assumed to take place within the SIN (Hanski et al., 2017). This allows for a certain degree of replication of metapopulations in a natural setting.

- Each of these 88 SINs is treated as a metapopulation, and represent a wide range of metapopulation structures. The number of habitat patches in the SINs ranges from 2 to 147, and patch sizes ranged from 0.001 to 10.2 hectares. Patch size within SINs tends to be quite variable, with coefficient of variation (mean divided by standard deviation) varying between 0.03 and 0.34 . This range of metapopulation structures provides both challenge and opportunity to examine the relationship between metapopulation capacity and persistence time distributions.

. $R$ code and data to reproduce the analyses is provided at https://doi.org/10.6084/m9.figshare.12576038.

\section{Metapopulation capacity: the structural persistence measure}

Metapopulation capacity estimates the ability of a metapopulation to support long-term persistence of a given species (Hanski and Ovaskainen, 2000) based on the distances among habitat patches in the spatial network. Specifically, metapopulation capacity $(\lambda)$ is the dominant eigenvalue of the landscape matrix $\mathbf{M}$, which is a square matrix describing dispersal connections among habitat patches. Concretely, the diagonal elements of the landscape matrix $\mathbf{M}$ are zero, and off-diagonal elements estimate dispersal probabilities between two habitat patches $i$ and $j$ that are some distance $d_{i j}$ away from one another. The landscape matrix $\mathbf{M}$ is estimated for each SIN, assuming that an exponential decay function as the basis for dispersal (Equation 1), based on previous research in this system (Hanski et al., 2017).

$$
\mathbf{M}=A_{i}^{x+\gamma} e^{-\epsilon d_{i j}} A_{j}^{\psi}
$$


. In the original formulation, entries of the landscape matrix $(\mathbf{M})$ were defined by including patch area $\left(A_{i}\right.$ and $\left.A_{j}\right)$ as a surrogate measure of carrying capacity. However, non-linear relationships and density-dependent dispersal probabilities may influence the relationship between patch area and population size (and subsequent dispersal probabilities). To address this, we formulate the $\mathbf{M}$ matrix with the inclusion of patch area, assuming that immigration $(\gamma)$, extinction $(x)$, and emigration $(\psi)$ are functions of patch area and collectively balance (i.e., $x+\gamma=\psi=0.25$; Hanski et al. (2017)). We examine the influence of excluding patch area in the calculation of the M matrix in the Supplemental Materials.

\section{Persistence time distributions: the temporal persistence measure}

We examine power law scaling relationships in persistence time distributions obtained for each semi-independent network (SIN). For a given SIN, we calculated persistence times for each patch over the course of the study period (1993-2016). Persistence was defined as any consecutive period that a given patch was occupied, taking values between 1 to 24 . While previous studies have developed approaches to address the potential left and right censoring of the time series data (i.e., patches may persist for longer than 24 years. Due to the extremely dynamic nature of the SINs examined - mean patch persistence time across SINs ranged from 1 to 4.8 - we do not attempt to extrapolate to unsampled periods. No patch was occupied for every sampling period, and only 4 out of the 2249 habitat patches in the 88 SINs examined persisted for 23 years.

- Because of the dynamic nature of these metapopulations, patches could contribute multiple persistence times to the distribution. This means that patches that go extinct and are recolonized contribute more data to the distribution. However, it is the persistent patches that drive the heavy tail of the persistence time distribution, as well as the corresponding value of $\alpha$. This $\alpha$ is estimated using maximum likelihood, following the equation

$$
\hat{\alpha}=1+n\left[\sum_{i=1}^{n} \log \left(\frac{x_{i}}{x_{\min }-0.5}\right)\right]^{-1}
$$


- Here, we use the hatted symbol $(\hat{\alpha})$ to denote $\alpha$ as estimated from data. The parameter $x_{\min }$ is the lower bound of persistence times $x$ where the power law can be fit to the data. Each SIN has a fit $x_{\min }$ and $\alpha$ value. In the Supplemental Materials, we explore the distribution and relationship between $x_{\min }$ and $\alpha$ values fit for each SIN. Power law distributions were fit using the poweRlaw package in $\mathrm{R}$, following the bootstraping procedure to account for parameter uncertainty (Gillespie, 2015). Further, goodness of fit to the power law distribution was determined via bootstrapping following Clauset et al. (2009).

\section{Relating metapopulation persistence measures}

We related metapopulation capacity $(\lambda)$ to the power law fit parameter $(\alpha)$ characterizing the tail of the persistence time distribution for each SIN using a Spearman's rank correlation to account for a potentially non-linear relationship. Larger values of metapopulation capacity are indicative of a greater chance of species persistence in the metapopulation. Larger values of persistence time fits $(\alpha)$ correspond to a faster decay in persistence times, and an increased number of short-lived but quickly recolonized patches. Assuming that consistently colonized patches are a sign of network-level persistence, a negative relationship between metapopulation capacity $(\lambda)$ and persistence time fits $(\alpha)$ is expected. However, if we interpret the rapid recolonization and extinction of patches as a signature of a dynamic, but persistent, metapopulation, a positive relationship may emerge.

\section{Correlates of metapopulation persistence measures}

Numerous environmental covariates may influence habitat patch quality, which affects subsequent colonization and extinction dynamics (Fleishman et al., 2002; Thomas, 1994). Given that metapopulation capacity does not directly incorporate information on variation in patch quality, but that the persistence time distributional fit does likely reflect patch quality, we would expect that environmental conditions would most strongly correlate with the persistence time distributional fits $(\alpha)$. 
- Patch area was estimated during sampling, with the median patch area being approximately 0.6 ha, and the majority of habitat patches smaller than 2 ha. The two common host plants of $M$. cinxia are Plantago lanceolata and Veronica spicata. We quantified resource availability as the sum of abundance of these two host plants based on an ordinal scale between 0 and 3 for each species, with larger values corresponding to a greater plant abundance (Ojanen et al., 2013). Previously, the summed abundance of these two host plants has been predictive of colonization, extinction, and occupancy in the Åland islands (Dallas et al., 2019). For each SIN, we calculated the mean resource abundance and the variance in resource abundance. Grazing pressure was estimated as the estimated fraction of the habitat patch subjected to grazing based on observations of damaged plants. Plantago lanceolata, which serves as the dominant host plant through much of the Åland island system, is infected by a powdery mildew pathogen (Podosphaera plantaginis; Tollenaere et al. (2014)), which reduces plant resource quality and subsequent overwintering survival and emergence of larvae in the spring (van Nouhuys and Laine, 2008). Mildew infection was estimated as the mean fraction of patches within each SIN where the mildew pathogen was present across each sampling period.

Aspects of the structure of each SIN may be related to metapopulation persistence. These include the number of habitat patches in the SIN, as well as several measures of spatial network structure. For instance, the tendency of patches to cluster into small groups, forming smaller communities in which dispersal is expected to be stronger, is likely related to spatial network persistence (Fletcher Jr et al., 2013). To quantify this, we used a series of measures which capture different aspects of community formation. All measures were performed on the weighted landscape matrix $M$ for each SIN, where weights were the dispersal probabilities generated from the negative exponential dispersal kernel described above.

- First, we estimated modularity of the network by first identifying clusters within each SIN using the random walk approach of Pons and Latapy (2005), and then quantifying the tendency of these identified communities to result in a modular network, estimated using the igraph $\mathrm{R}$ package (Csardi and Nepusz, 2006). Second, we calculated the hub score of the landscape matrix 
$M$ (Kleinberg, 1999), which is nearly identical to calculation as metapopulation capacity, and is the dominant eigenvalue corresponding to the principal eigenvector of $M \times t(M)$ (the landscape matrix $M$ multiplied by its transpose). Lastly, we measured a weighted form of transitivity also referred to as the 'clustering coefficient' - which quantifies the degree of spatial aggregation in habitat patches within a given SIN (Barrat et al., 2007). All of these measures attempt to address the distribution of patches in each SIN with respect to their estimated dispersal links estimated in Equation 1. Consequently, it is important to note that estimates of network structure described above will be sensitive to the formation of the landscape matrix $(M)$. Given that only metapopulation capacity $(\lambda)$ uses information contained in the landscape matrix $(M)$, we would expect metapopulation capacity to be more strongly related to these aspects of dispersal network structure than persistence time distributional fits.

\section{Results}

Some SINs $(n=27)$ did not have enough data to compute distributional fits to the persistence times $(\alpha)$. For the SINs that did have enough data, the best fit values of $x_{\min }$ and $\alpha$ were quite variable (see Supplemental Material, Figures A2 - A4). The majority of $\alpha$ values were between 2 and 3, supporting previous observations (Clauset et al., 2009). Based on bootstrap tests, there is evidence that the power law is the best fit distribution for $87 \%$ ( $n=53$ of the 61 SINs) of the persistence time distributions, based on a significance level of 0.05 following the procedure of Clauset et al. (2009). The $p$-value generated from this test can be used as a measure of plausibility of the fit between empirical data and power law fit. It is not a test of the goodness-of-fit of the power law directly, as $p>0.05$ cannot be interpreted as support of the power law fit, while $p<$ 0.05 would suggest that the power law is not the best fit.

- There was no apparent spatial pattern in power law fit parameter $(\alpha)$ to the persistence time distribution (Figure 1d) or metapopulation capacity (Figure 1b) of each SIN, though clear variation was observed in both fit persistence time distributions $(\alpha \in[1.86-5.04])$ and metapopulation capacities $(\lambda \in[0.003-1.56])$. Further, there was no significant relationships observed between 
either metapopulation persistence measure - metapopulation capacity $(\lambda)$ or persistence time distributional fits $(\alpha)$ - to either mean patch persistence times or mean fraction of occupied patches (Figure 2). However, the two measures of metapopulation persistence were strongly related to one another (Figure 3), suggesting a clear link between the two measures of metapopulation persistence.

\section{Correlates of metapopulation persistence measures}

We then related a set of environmental (e.g., mean resource availability) and network (e.g., number of habitat patches) to both persistence time distributions $(\alpha)$ and metapopulation capacities $(\lambda)$ for each of the studied SINs. We hypothesized that variables not captured in the landscape matrix may be better described by persistence time distributions, while structural properties of the landscape matrix $(M)$ may be more strongly related to metapopulation capacity. We found that environmental variables and measures of spatial network structure tended to be more strongly related to metapopulation capacity (Figure 4). The exception to this was the mean fraction of patches infected by a mildew pathogen, which was negatively related to persistence time distributional fits, while we failed to detect any relationship with metapopulation capacity (Figure 4). Together, we found strong relationships between the metapopulation capacity and both measures of dispersal network structure and local environmental covariates (Figure 4), but generally slightly weaker relationships for the distributional fits to patch persistence times.

\section{Discussion}

The majority of patch persistence time distributions were best fit by the power law, clarifying a link between extinction time distributions from population ecology - as well as other power law relationships (Marquet et al., 2005) - and patch-scale persistence time distributions of interconnected populations. Weak correlations between composite measures of each SIN (mean persistence time and mean occupancy) and metapopulation capacity belie the significant positive relationship between structural (metapopulation capacity) and temporal (persistence time distributional fit) 
measures. This provides evidence for a clear relationship between the two measures of metapopulation persistence, despite the two measures using information on either static network topology (as estimated in the landscape matrix $M$ ) or temporal data on patch persistence times, effectively linking two approaches to the estimation of metapopulation persistence. Further, several environmental and network structural variables were correlated with both metapopulation persistence measures. However, we found little support for the hypothesis that persistence time distributional fits would be more closely related to aspects of patch quality which are not considered in the calculation of metapopulation capacity. This suggests that - at least in the Åland island system - persistence time distributions for each SIN are largely unrelated to habitat variables at the scale of the entire metapopulation. Taken together, this suggests a strong link between spatial network topology and the resulting dynamics, provides evidence for the use of persistence time distributions to understand metapopulation persistence, and extends theory related to heavy-tailed population extinction time distributions to understanding interconnected populations and metapopulations.

- Patch persistence time distributions characterized by high values - corresponding to dynamic metapopulations where rapid colonization and extinction events shorten the tail of the persistence time distribution - were associated with high metapopulation capacity $(\alpha)$. This suggests that the existence of long-term persistent patches may not be a signature of overall metapopulation persistence. The opposite appears to be the case, where metapopulations composed of patches which rapidly become extinct and are rapidly recolonized tend to be the most structurally persistent (based on metapopulation capacity). This finding may be influenced by species traits such as dispersal ability and survival. However, a species which colonizes a set of habitat patches and persists in each patch is not a true metapopulation (Harrison and Taylor, 1997). However, the persistence time distribution may be useful outside of these true metapopulations, as understanding the distribution of extinction times is central to population ecology (Drake, 2006, 2014). Further, the persistence time distribution may signal metapopulation "type" (as defined in Harrison and Taylor (1997)), as mainland-island metapopulations would have a longer tailed persistence time distribution relative to the classical metapopulation or patchy population. 
We failed to detect strong relationships between persistence time distributional fits and local-scale environmental variation in the Åland island metapopulation. The lack of relationship between persistence time distributional fits and patch quality variables might simply be a function of the inherent variation in persistence time distributions and the subsequent power law distributional fits. This is because habitat patch persistence may largely be a stochastic process, in which patches go extinct and are recolonized often. This, in turn, strongly influences the distribution of persistence times and resulting distributional fits. Despite the weak relationships between patch quality and persistence time distributions, we found strong relationships between network structure (e.g., modularity) and both measures of metapopulation persistence, suggesting a signal of the effect of landscape matrix structure on resulting metapopulation persistence. Lastly, metapopulation capacity was found to be positively related to local-scale habitat covariates (e.g., mean patch area), even when patch area was not used to quantify dispersal links in the metapopulation (Figure A8). These correlations could not be explained by the associations between patch area, resource abundance, and grazing pressure (Figure A9) alone (see Supplemental Materials for further discussion). Spatial autocorrelation in local environmental conditions which scale up to the network level might result in correlations between environmental covariates and metapopulation capacity as well. Examining other metapopulation systems may provide insight into the relative strength of relationships between environmental and topological covariates and measures of metapopulation persistence.

. To date, metapopulation persistence in a dynamic sense has largely been determined through model simulations, which quantify metapopulation persistence as the fraction of simulations in which the metapopulation avoids extinction (Molofsky and Ferdy, 2005) or the mean time until metapopulation extinction (Johst et al., 2002). While models may be parameterized with observational data, there remains a disconnect between the theory of metapopulation persistence and metapopulation dynamics in natural systems (Moilanen, 2002). By quantifying metapopulation persistence using the distribution of persistence times, it is possible to characterize metapopulation persistence without the necessity of metapopulation extinction. However, the fit power law parameter $(\alpha)$ to the distribution of persistence times has some limitations. For instance, imperfect 
detection could cause gaps that strongly influence the tail of the persistence time distribution (i.e., those long persisting patches), which can alter the $\alpha$ parameter of the power law. Further, the habitat patches which go extinct and are recolonized differentially contribute to the distribution of persistence times, as they can contribute many small values, whereas persistent patches contribute fewer values. The ideal measure of metapopulation persistence would incorporate both information on the spatial arrangement of habitat patches and the persistence times of patches. Currently, the measures of metapopulation persistence examined here rely on either spatial patch arrangement (metapopulation capacity) or patch persistence times (power law fits). Future work should attempt to bridge this gap to capture a complete view of metapopulation persistence, as well as incorporating the role of self-connections of habitat patches (Zamborain-Mason et al., 2017). Lastly, it is noteworthy that these measures of metapopulation persistence may be independent of metapopulation stability in some situations. That is, the measures of metapopulation persistence used here may not capture the ability of the metapopulation to recover from a perturbation (Gilarranz et al., 2017) (but see Ovaskainen and Hanski (2002)) or targeted attack (Albert et al., 2000).

The relationship between spatial dispersal network structure and resulting metapopulation dynamics is not only of theoretical interest. Designing reserves capable of sustaining persistent populations is a high priority in conservation biology and management of endangered species (McCarthy et al., 2004; Nicholson and Ovaskainen, 2009). For the majority of these systems, the data necessary to calculate persistence time dsitributions are not available. Thus, the finding of a positive relationship between structural measures of metapopulation persistence and their temporal counterparts suggests that the use of spatial habitat patch arrangement in reserve design is justified as a means to enhance metapopulation persistence. Beyond reserve design, the arrangement of nodes in spatial networks in a fashion that maximizes persistence is of great importance to the design of many different types of networks (Ebel et al., 2002; Kamra et al., 2006; Rothenberg, 2001; Wu et al., 2017), including those related to transportation (e.g., highways), communication (e.g., telephone service centers), disease transmission, and sensor arrays (e.g., air quality towers). Providing demonstrations of the relationships between topological properties of 
networks and their corresponding dynamics will further aid the creation of persistent networks.

Identifying these topological properties in ecological networks provides evidence for self-organization to promote persistence, providing insight into the structure and stability of ecological systems. 
Albert R, Jeong H, Barabási AL (2000) Error and attack tolerance of complex networks. nature 406(6794):378

Barrat A, Barthelemy M, Vespignani A (2007) The architecture of complex weighted networks: Measurements and models. In: Large Scale Structure And Dynamics Of Complex Networks: From Information Technology to Finance and Natural Science, World Scientific, pp 67-92

van Bergen E, Dallas T, DiLeo MF, Kahilainen A, Mattila AL, Luoto M, Saastamoinen M (2020) The effect of summer drought on the predictability of local extinctions in a butterfly metapopulation. Conservation Biology

Bertuzzo E, Suweis S, Mari L, Maritan A, Rodríguez-Iturbe I, Rinaldo A (2011) Spatial effects on species persistence and implications for biodiversity. Proceedings of the National Academy of Sciences

Chen S, ODea EB, Drake JM, Epureanu BI (2019) Eigenvalues of the covariance matrix as early warning signals for critical transitions in ecological systems. Scientific reports 9(1):1-14

Clauset A, Shalizi CR, Newman ME (2009) Power-law distributions in empirical data. SIAM review 51(4):661-703

Csardi G, Nepusz T (2006) The igraph software package for complex network research. InterJournal Complex Systems:1695, URL http:/ /igraph.org

Dallas TA, Saastamoinen M, Schulz T, Ovaskainen O (2019) The relative importance of local and regional processes to metapopulation dynamics. Journal of Animal Ecology 89(3)

Diekmann O, Heesterbeek J, Roberts MG (2010) The construction of next-generation matrices for compartmental epidemic models. Journal of the Royal Society Interface 7(47):873-885

Doebeli M (1995) Dispersal and dynamics. Theoretical population biology 47(1):82-106

Drake JM (2006) Extinction times in experimental populations. Ecology 87(9):2215-2220 
Drake JM (2014) Tail probabilities of extinction time in a large number of experimental populations. Ecology 95(5):1119-1126

Ebel H, Mielsch LI, Bornholdt S (2002) Scale-free topology of e-mail networks. Physical review E 66(3):035103

Fleishman E, Ray C, Sjögren-Gulve P, Boggs CL, Murphy DD (2002) Assessing the roles of patch quality, area, and isolation in predicting metapopulation dynamics. Conservation Biology 16(3):706-716

Fletcher Jr RJ, Revell A, Reichert BE, Kitchens WM, Dixon JD, Austin JD (2013) Network modularity reveals critical scales for connectivity in ecology and evolution. Nature communications 4:2572

Gilarranz LJ, Rayfield B, Liñán-Cembrano G, Bascompte J, Gonzalez A (2017) Effects of network modularity on the spread of perturbation impact in experimental metapopulations. Science 357(6347):199-201

Gillespie CS (2015) Fitting heavy tailed distributions: The poweRlaw package. Journal of Statistical Software 64(2):1-16, URL http:/ /www.jstatsoft.org/v64/i02/

Hanski I (1994) A practical model of metapopulation dynamics. Journal of animal ecology pp $151-162$

Hanski I (1999) Metapopulation Ecology. Oxford Series in Ecology and Evolution, OUP Oxford, England

Hanski I (2011) Habitat loss, the dynamics of biodiversity, and a perspective on conservation. Ambio 40(3):248-255

Hanski I, Gilpin M (1991) Metapopulation dynamics: brief history and conceptual domain. In: Metapopulation dynamics: Empirical and theoretical investigations, Elsevier, pp 3-16

Hanski I, Ovaskainen O (2000) The metapopulation capacity of a fragmented landscape. Nature 404(6779):755 
Hanski I, Thomas CD (1994) Metapopulation dynamics and conservation: a spatially explicit model applied to butterflies. Biological Conservation 68(2):167-180

Hanski I, Schulz T, Wong SC, Ahola V, Ruokolainen A, Ojanen SP (2017) Ecological and genetic basis of metapopulation persistence of the glanville fritillary butterfly in fragmented landscapes. Nature Communications 8:14504

Harrison S, Taylor AD (1997) Empirical evidence for metapopulation dynamics. In: Metapopulation biology, Elsevier, pp 27-42

Holland MD, Hastings A (2008) Strong effect of dispersal network structure on ecological dynamics. Nature 456(7223):792

Holmes CJ, Rapti Z, Pantel JH, Schulz KL, Cáceres CE (2020) Patch centrality affects metapopulation dynamics in small freshwater ponds. Theoretical Ecology pp 1-14

Johst K, Brandl R, Eber S (2002) Metapopulation persistence in dynamic landscapes: the role of dispersal distance. Oikos 98(2):263-270

Kamra A, Misra V, Feldman J, Rubenstein D (2006) Growth codes: Maximizing sensor network data persistence. In: ACM SIGCOMM Computer Communication Review, ACM, vol 36, pp 255-266

Kéfi S, Rietkerk M, Alados CL, Pueyo Y, Papanastasis VP, ElAich A, De Ruiter PC (2007) Spatial vegetation patterns and imminent desertification in mediterranean arid ecosystems. Nature 449(7159):213-217

Keitt TH, Stanley HE (1998) Dynamics of north american breeding bird populations. Nature 393(6682):257-260

Keymer JE, Marquet PA, Velasco-Hernández JX, Levin SA (2000) Extinction thresholds and metapopulation persistence in dynamic landscapes. The American Naturalist 156(5):478-494

Kleinberg JM (1999) Authoritative sources in a hyperlinked environment. Journal of the ACM (JACM) 46(5):604-632 
Kleinhans D, Jonsson PR (2011) On the impact of dispersal asymmetry on metapopulation persistence. Journal of theoretical biology 290:37-45

Levins R (1969) Some demographic and genetic consequences of environmental heterogeneity for biological control. American Entomologist 15(3):237-240

Marquet PA, Quiñones RA, Abades S, Labra F, Tognelli M, Arim M, Rivadeneira M (2005) Scaling and power-laws in ecological systems. Journal of Experimental Biology 208(9):1749-1769

Martín HG, Goldenfeld N (2006) On the origin and robustness of power-law species-area relationships in ecology. Proceedings of the National Academy of Sciences 103(27):10310-10315

McCarthy MA, Thompson CJ, Possingham HP (2004) Theory for designing nature reserves for single species. The American Naturalist 165(2):250-257

McCullough DR (1996) Metapopulations and wildlife conservation. Island Press

Moilanen A (2002) Implications of empirical data quality to metapopulation model parameter estimation and application. Oikos 96(3):516-530

Molofsky J, Ferdy JB (2005) Extinction dynamics in experimental metapopulations. Proceedings of the National Academy of Sciences 102(10):3726-3731

Morse D, Lawton J, Dodson M, Williamson M (1985) Fractal dimension of vegetation and the distribution of arthropod body lengths. Nature 314(6013):731-733

Nicholson E, Ovaskainen O (2009) Conservation prioritization using metapopulation models. Spatial conservation prioritisation: quantitative methods and computational tools Oxford University Press, Oxford, UK pp 110-121

van Nouhuys S, Laine AL (2008) Population dynamics and sex ratio of a parasitoid altered by fungal-infected diet of host butterfly. Proceedings of the Royal Society B: Biological Sciences 275(1636):787-795 
Ojanen SP, Nieminen M, Meyke E, Pöyry J, Hanski I (2013) Long-term metapopulation study of the glanville fritillary butterfly (melitaea cinxia): survey methods, data management, and long-term population trends. Ecology and Evolution 3(11):3713-3737

Ovaskainen O, Hanski I (2001) Spatially structured metapopulation models: global and local assessment of metapopulation capacity. Theoretical Population Biology 60(4):281-302

Ovaskainen O, Hanski I (2002) Transient dynamics in metapopulation response to perturbation. Theoretical Population Biology 61(3):285-295

Ovaskainen O, Hanski I (2003) How much does an individual habitat fragment contribute to metapopulation dynamics and persistence? Theoretical Population Biology 64(4):481-495

Pons P, Latapy M (2005) Computing communities in large networks using random walks. In: International symposium on computer and information sciences, Springer, pp 284-293

Rothenberg R (2001) How a net works: implications of network structure for the persistence and control of sexually transmitted diseases and hiv. Sexually transmitted diseases 28(2):63-68

Saha S, Adiga A, Prakash BA, Vullikanti AKS (2015) Approximation algorithms for reducing the spectral radius to control epidemic spread. In: Proceedings of the 2015 SIAM International Conference on Data Mining, SIAM, pp 568-576

Staniczenko PP, Kopp JC, Allesina S (2013) The ghost of nestedness in ecological networks. Nature communications $4(1): 1-6$

Thomas C (1994) Extinction, colonization, and metapopulations: environmental tracking by rare species. Conservation Biology 8(2):373-378

Tollenaere C, Pernechele B, Mäkinen H, Parratt S, Németh M, Kovács G, Kiss L, Tack A, Laine AL (2014) A hyperparasite affects the population dynamics of a wild plant pathogen. Molecular ecology 23(23):5877-5887

Visconti P, Elkin C (2009) Using connectivity metrics in conservation planning-when does habitat quality matter? Diversity and Distributions 15(4):602-612 
Vuilleumier S, Bolker BM, Lévêque O (2010) Effects of colonization asymmetries on metapopulation persistence. Theoretical population biology 78(3):225-238

White EP, Enquist BJ, Green JL (2008) On estimating the exponent of power-law frequency distributions. Ecology 89(4):905-912

Wu Y, Shindnes G, Karve V, Yager D, Work DB, Chakraborty A, Sowers RB (2017) Congestion barcodes: Exploring the topology of urban congestion using persistent homology. In: Intelligent Transportation Systems (ITSC), 2017 IEEE 20th International Conference on, IEEE, pp 1-6

Zamborain-Mason J, Russ GR, Abesamis RA, Bucol AA, Connolly SR (2017) Network theory and metapopulation persistence: incorporating node self-connections. Ecology letters 20(7):815-831 
Figure 1: Estimates of metapopulation persistence were based on either the structure of the interaction network (a) or the distribution of patch persistence times (c), where measures exclusively consider either landscape matrix structure or patch persistence times, respectively. Estimates of metapopulation persistence are mapped onto the set of 88 semi-independent networks in the Åland islands ( $\mathbf{b}$ and $\mathbf{d}$ ), illustrating the variation in metapopulation capacity in $\mathbf{b}$ and the power law fit $(\alpha)$ to the persistence time distribution (d). Grey shaded polygons correspond to networks where network statistics could not be calculated.

Figure 2: Relationships between metapopulation persistence measures - metapopulation capacity $(\lambda)$ and persistence time distributions (power law fits; $\alpha$ ) - and the mean persistence time of patches (a and $\mathbf{b}$ ) and the mean fraction of occupied patches (c and $\mathbf{d}$ ) for each semi-independent network (SIN). Reported statistics correspond to Spearman's partial rank correlation coefficient and associated p-value. 
Figure 3: The relationship between metapopulation capacity $(\lambda)$ and persistence time distributions (power law fits; $\alpha$ ) for each semi-independent network (SIN). Error bars represent the estimated standard deviation in the $\alpha$ parameter, and point size is proportional to the $p$-value of the goodness of fit test for the power law fit to the persistence time distribution. Reported statistics correspond to Spearman's rank correlation coefficient and associated $p$-value.

Figure 4: Spearman's correlation coefficients between network (in blue) and environmental (in green) covariates, and both measures of metapopulation persistence ((metapopulation capacity $\lambda$ and persistence time distribution fits $\alpha$ ). The grey line corresponds to an equally strong correlation with both measures of metapopulation persistence. The majority of covariates are close to this line, signaling a similar relationship between each covariate and the two persistence measures. 
Figures
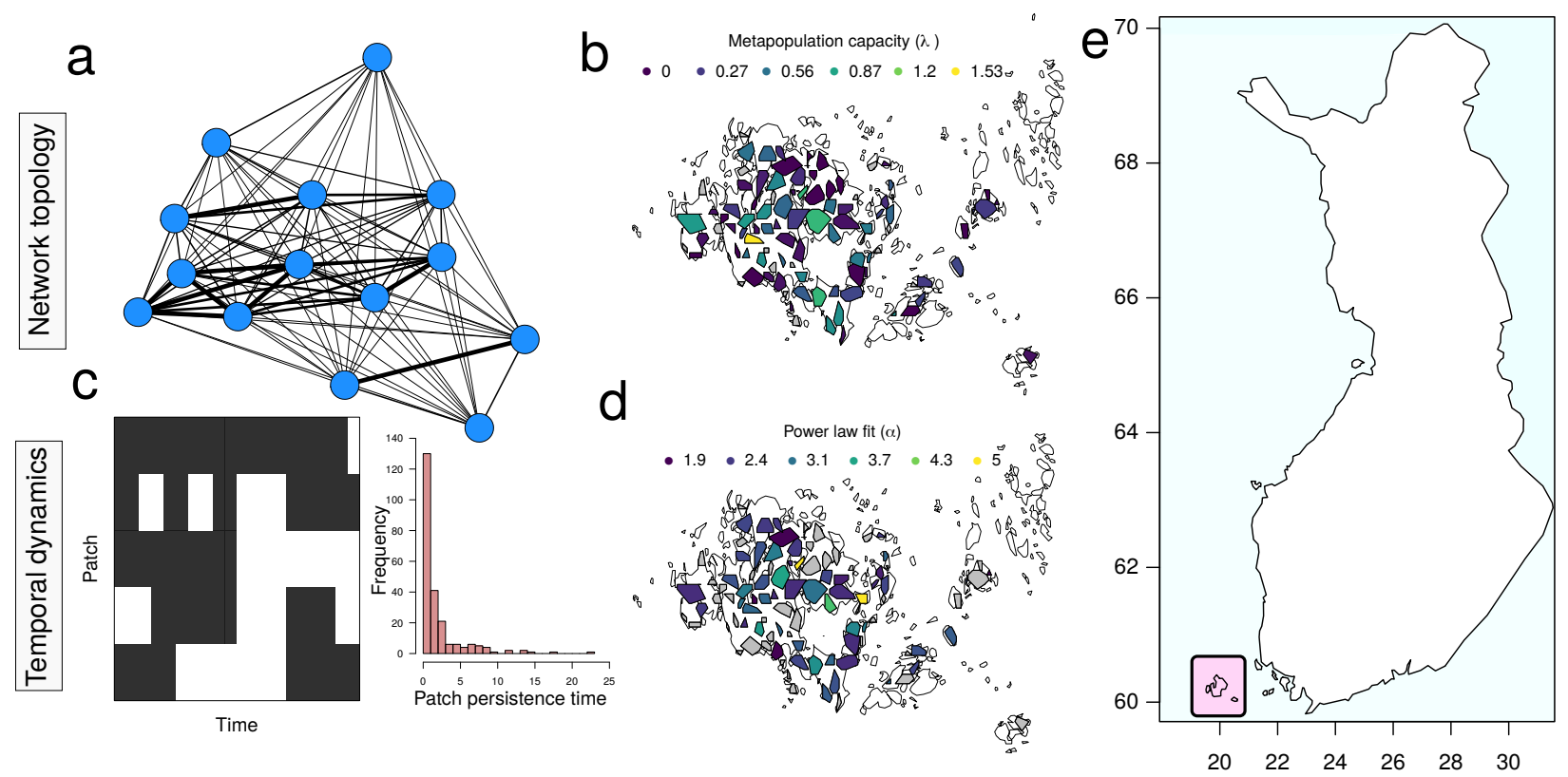

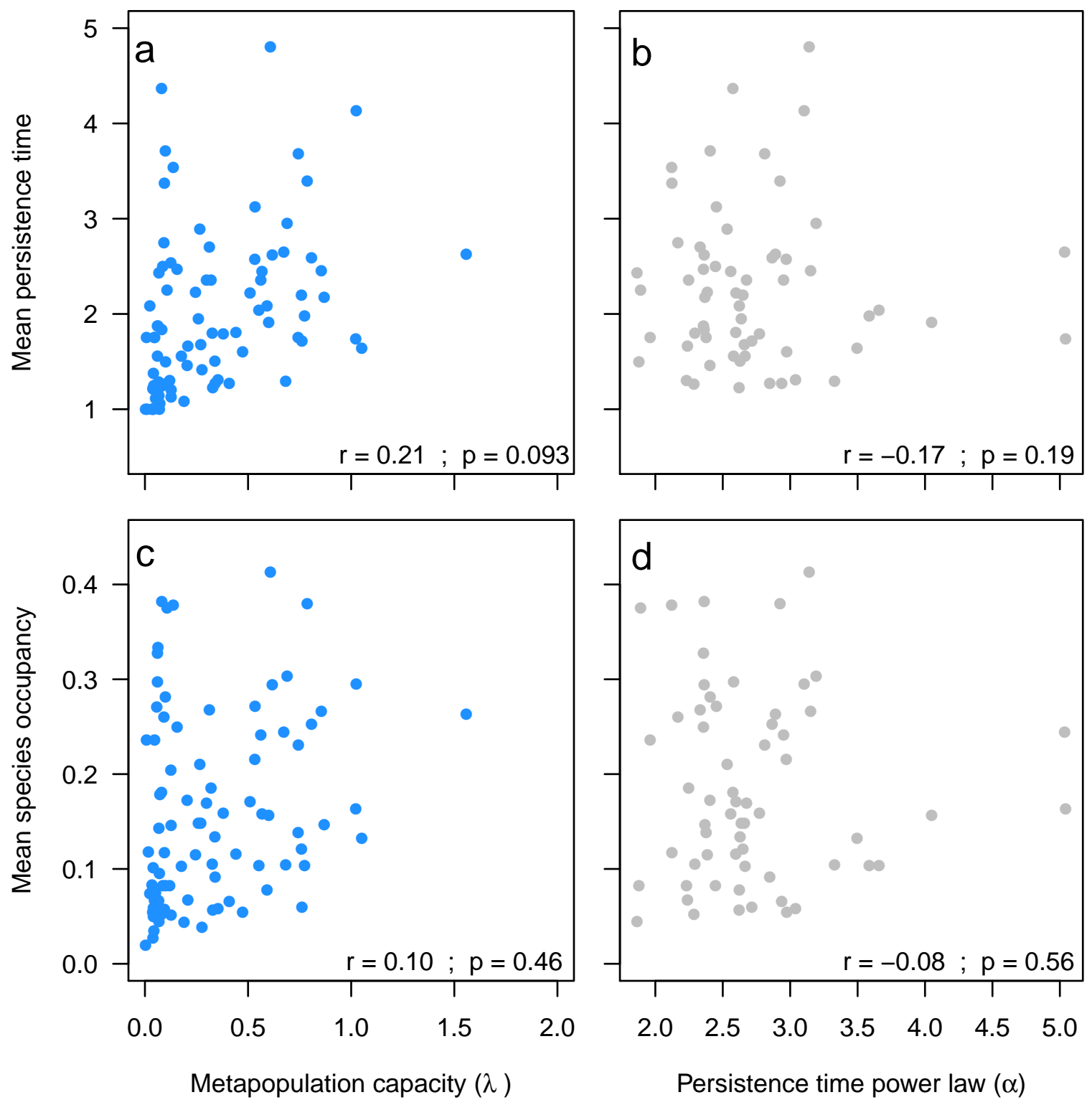


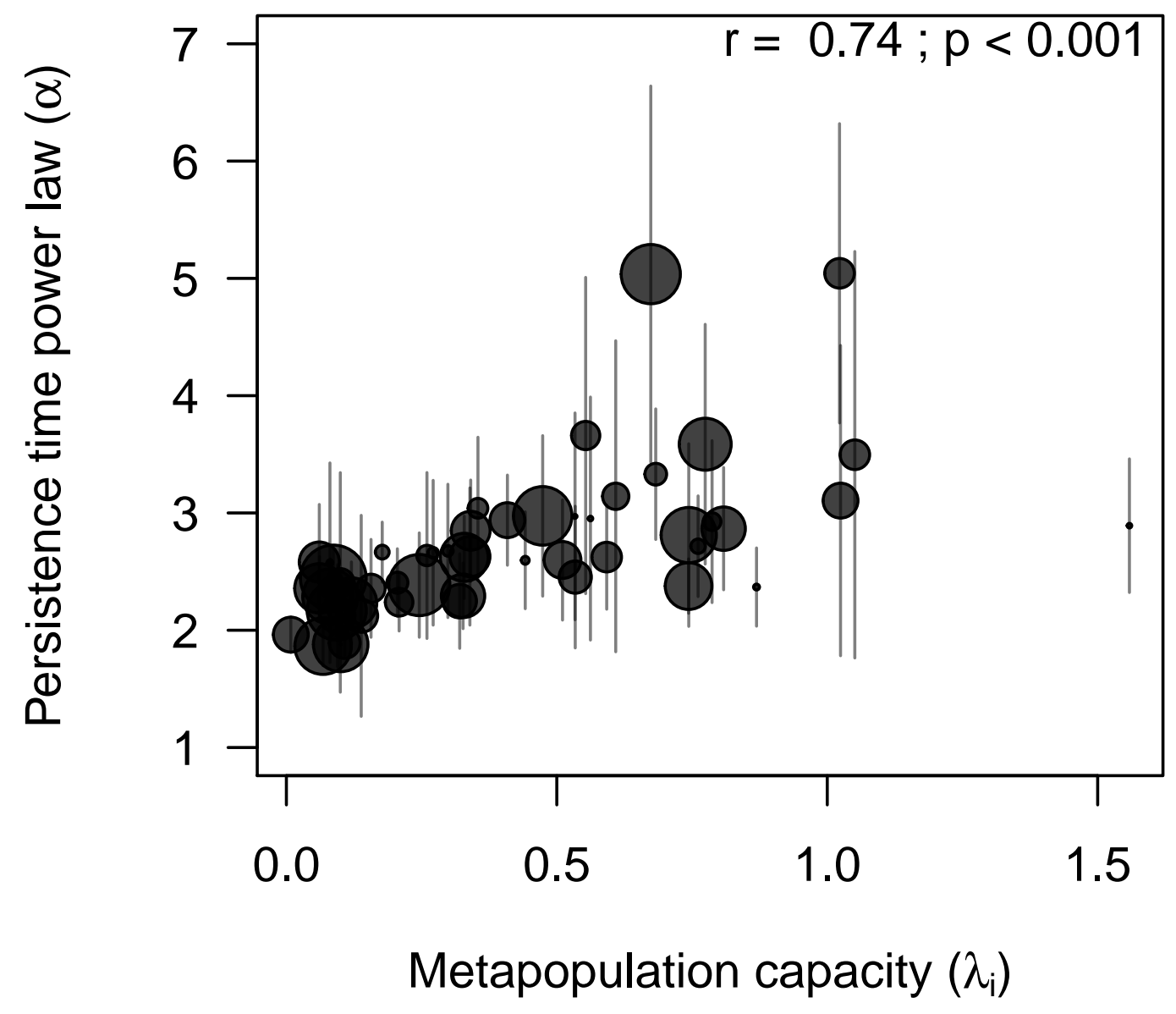




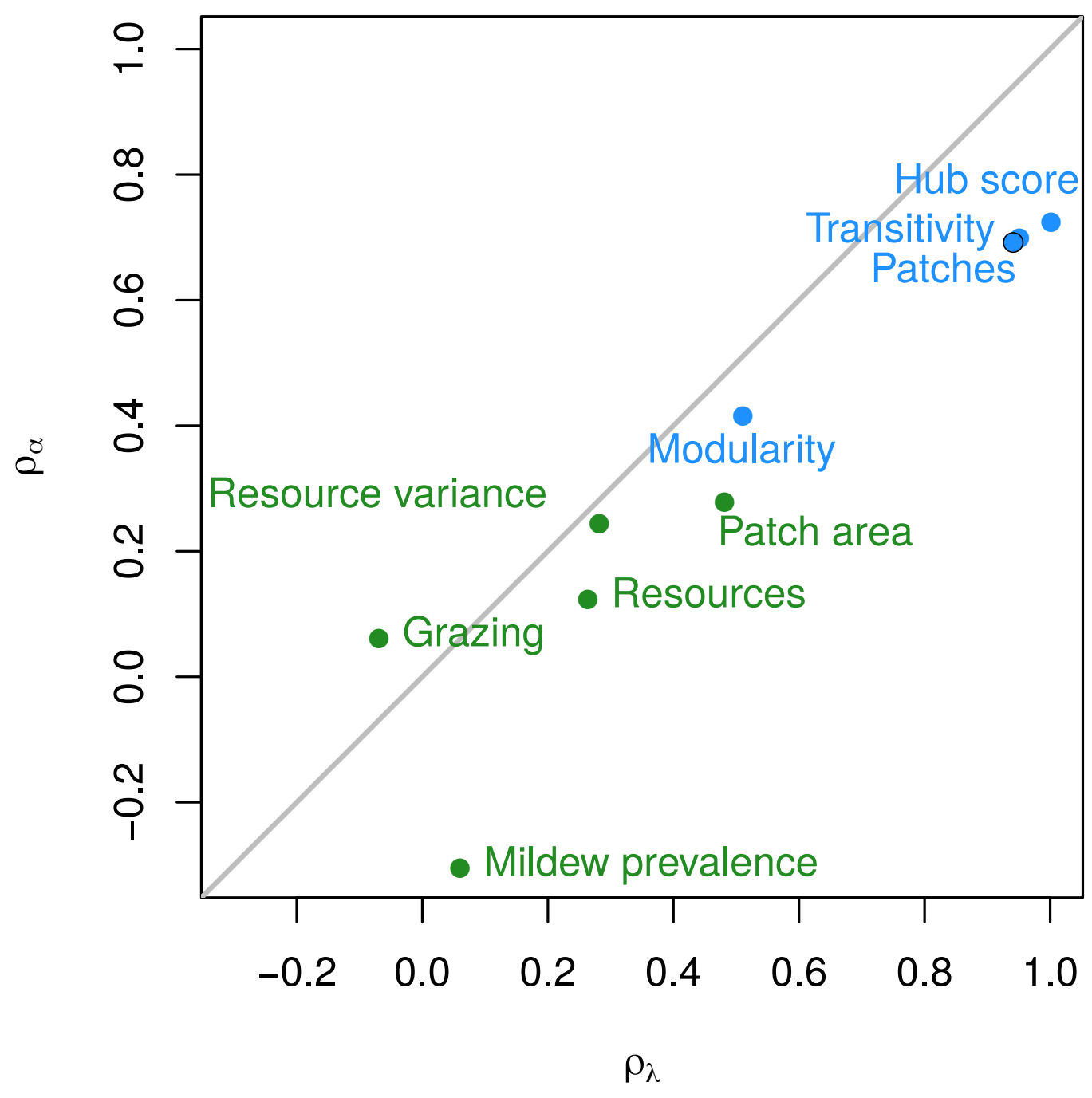


529

530

531

Tad Dallas $^{a, b, *}$, Marjo Saastamoinen ${ }^{b, c}$, Otso Ovaskainen ${ }^{b, d}$

${ }^{a}$ Department of Biological Sciences, Louisiana State University, Baton Rouge, LA, USA

${ }^{b}$ Organismal and Evolutionary Biology Research Programme, P.O. Box 65, 00014 University of Helsinki, Finland

${ }^{c}$ Helsinki Institute of Life Science, University of Helsinki, Finland

${ }^{d}$ Centre for Biodiversity Dynamics, Department of Biology, Norwegian University of Science and Technology. N-7491 Trondheim, Norway

* tad.a.dallas@gmail.com

The semi-independent networks (SINs) examined in this manuscript were made up of a wide range of habitat patches in terms of overall number of patches per SIN (Figure A1) and area of each patch (Figure A6). We view this as an overall strength, as this likely increases the chances that our findings are robust to other metapopulation systems. 


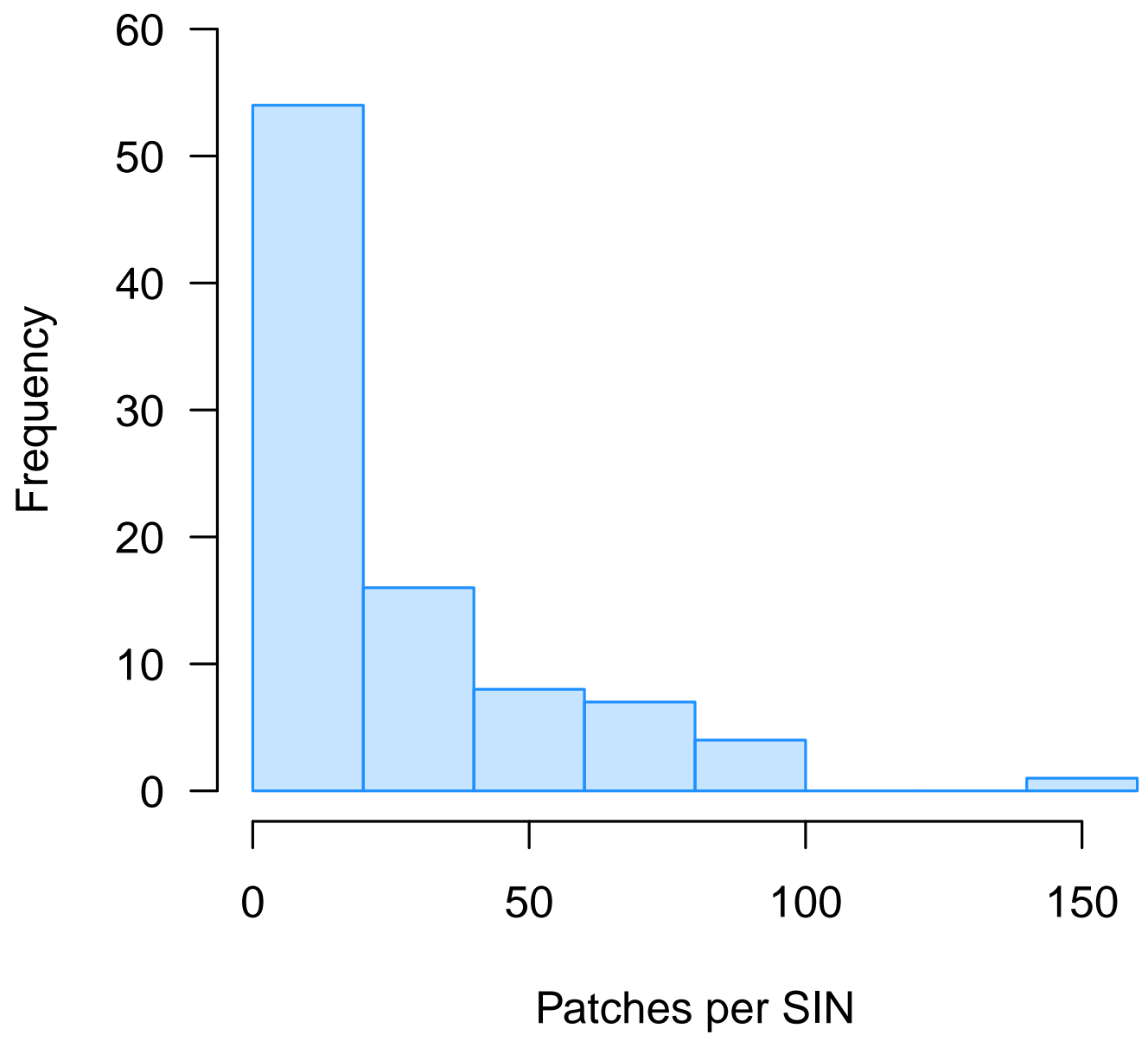

Figure A1: The distribution of the number of habitat patches for each semi-independent network (SIN) in the Åland islands system. 


\section{Power law fits to patch persistence times}

Best fit power law distributions to the empirical data different greatly in their best fit parameterizations of $x$ min - corresponding to the lower threshold persistence time in the power law fit (Figure A2)and $\alpha$-corresponding to the scaling or shape parameter of the power law (Figure A3). Further, the standard deviation in the best fit parameter for each semi-independent network tended to be fairly large as xmin and $\alpha$ became larger (Figure A4), demonstrating a clear relationship between the two fit parameters. Finally, the result of the best fit power laws to the persistence time distribution for a sample of the semi-independent networks demonstrates both the difficulty in fitting some distributions (e.g., SIN 107 in Figure A5) and the qualitative goodness of fit to other persistence time distributions (e.g., SIN 17,22, and 3 in Figure A5). 


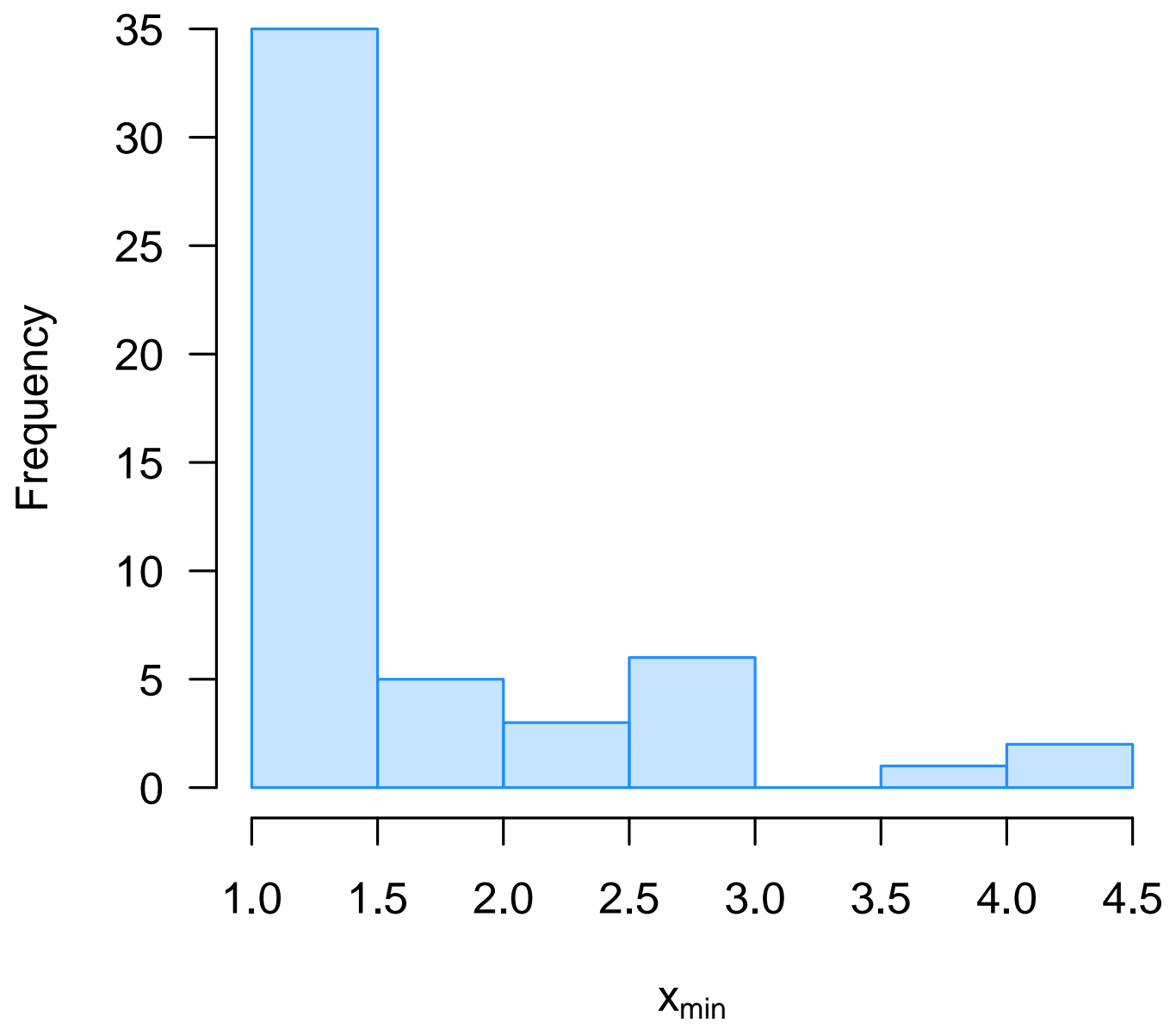

Figure A2: The distribution of $x$ min values for the set of semi-independent networks, corresponding to the lower threshold for the power law fitting procedure, optimized using the Kolmogorov-Smirnoff statistic. 


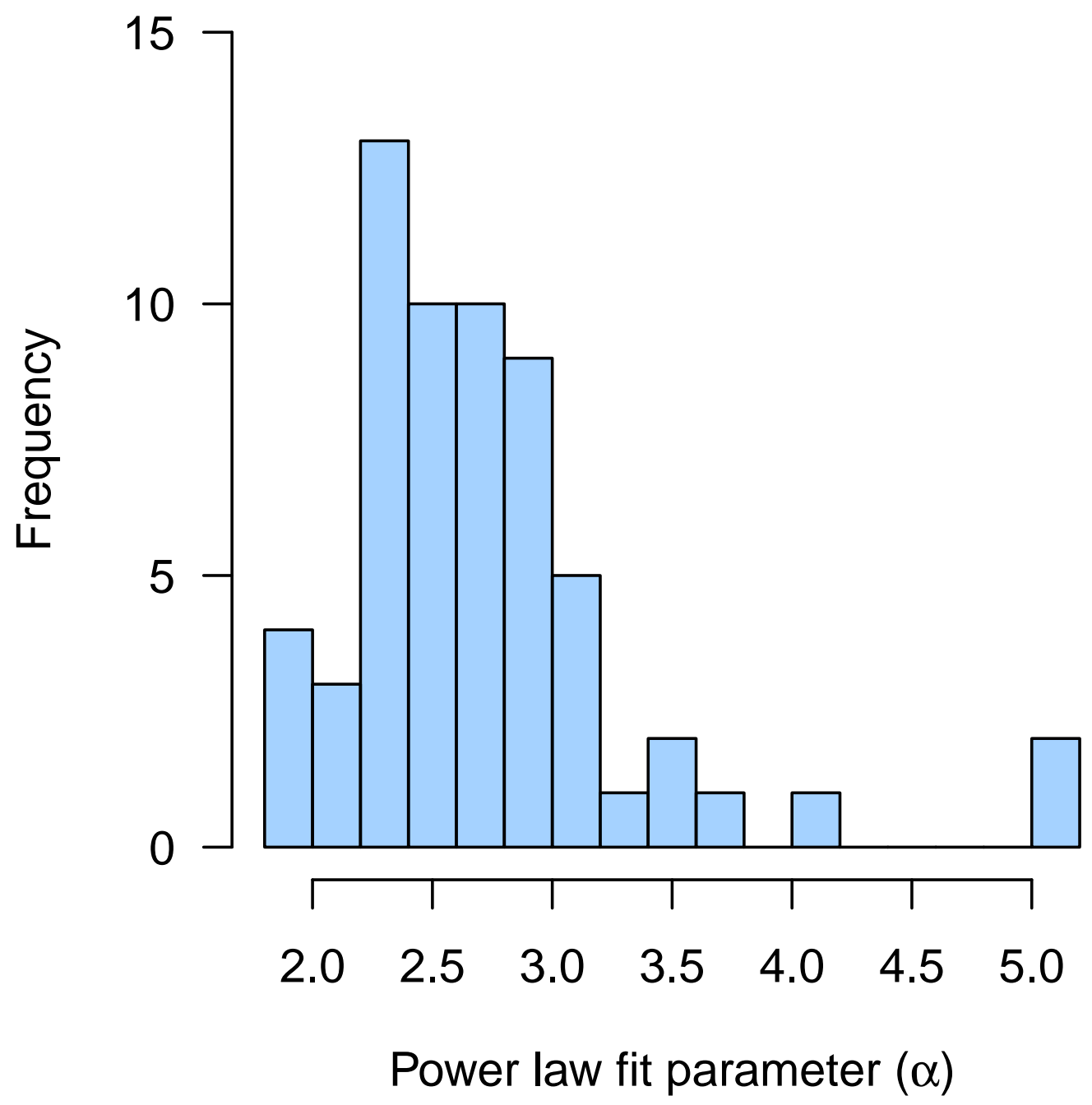

Figure A3: The distribution of power law fit scaling parameter $(\alpha)$ for the set of semi-independent networks. The majority of values fall between 2 and 3, as suggested in other empirical systems (Clauset et al., 2009). 


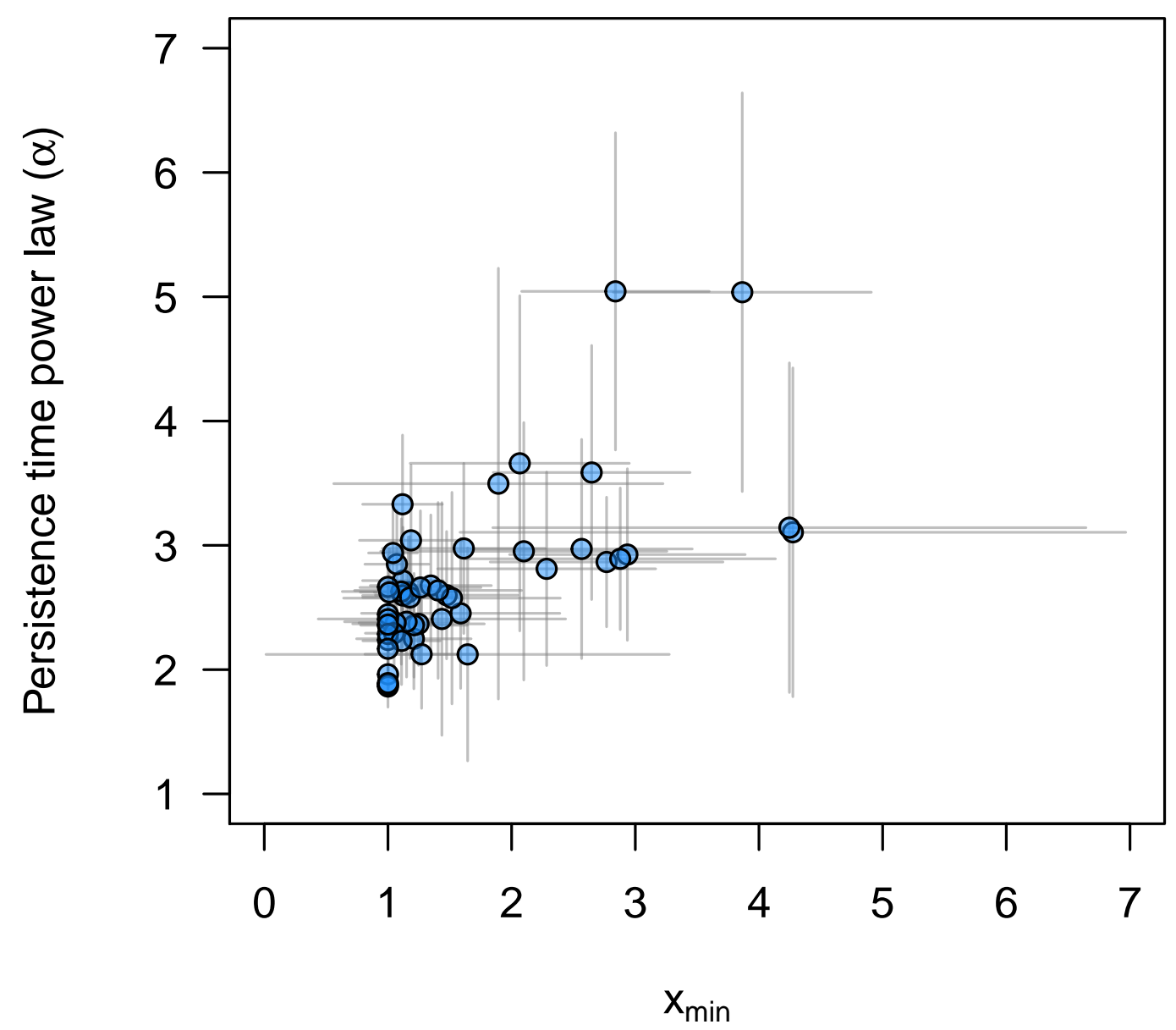

Figure A4: The relationship between the power law fit xmin and scaling parameter $(\alpha)$. Points are best fit values, and error bars correspond to standard deviations in parameter estimates. 

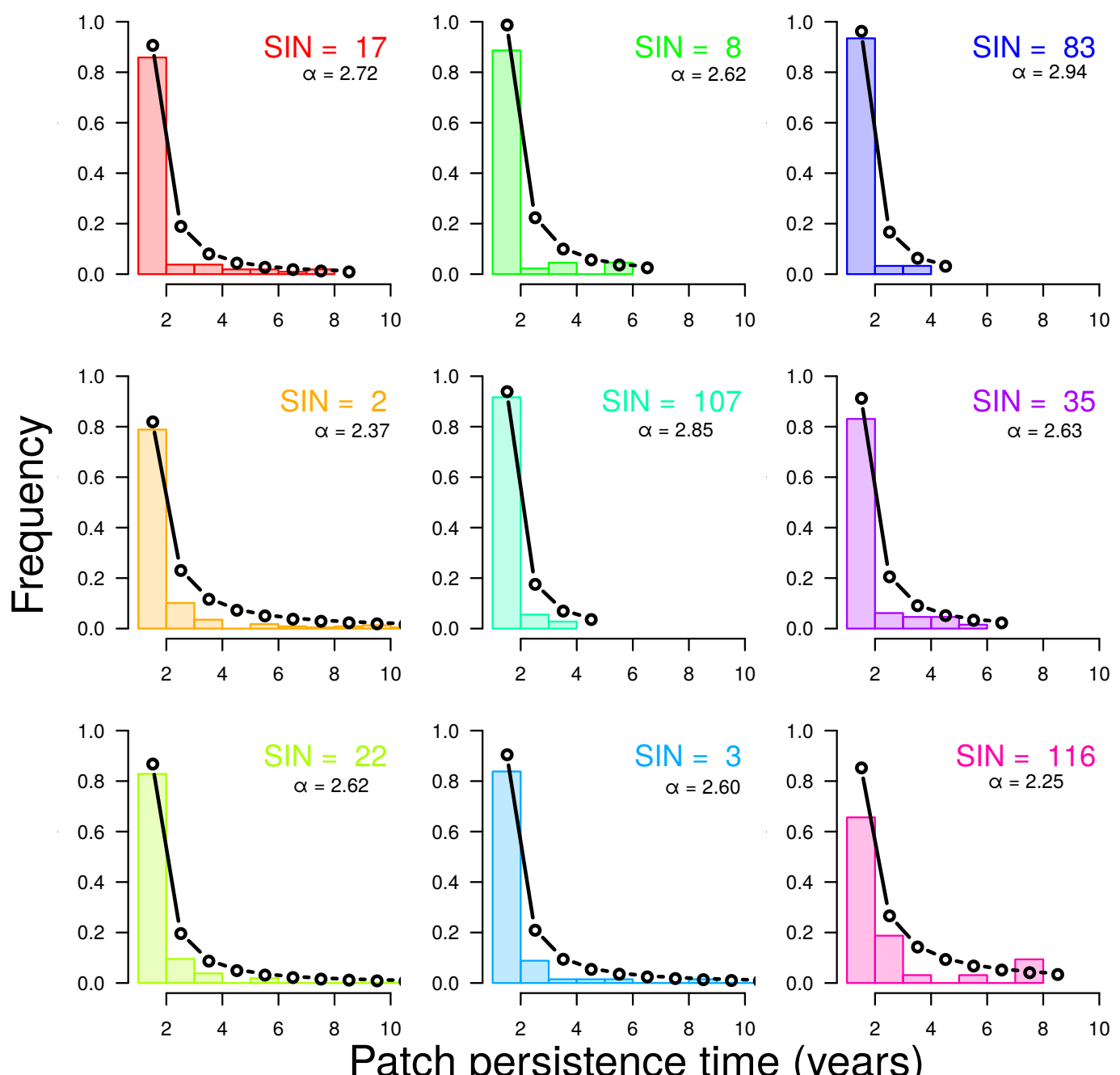

Figure A5: A set of nine of the patch persistence time distributions (bars) and power law fits (black lines, values of power law $\alpha$ parameter in black), where each panel corresponds to a single SIN (SIN identifier in color). 


\section{Without considering patch area in the landscape matrix}

Patch area was included in quantification of links between habitat patches. This assumes that larger habitat patches are more strongly connected than smaller habitat patches. The putative mechanism underlying this is that larger habitat patches support larger populations, and dispersal is density-dependent, resulting in a larger number of emigrants from large habitat patches. Patch area was quite variable in the set of habitat patches and SINs examined (Figure A6), suggesting that patch area may play a large role in estimating entries of the landscape matrix M. Here, we quantify metapopulation capacity based on a landscape matrix $(\mathbf{M})$ without the influence of patch area, finding strikingly similar results compared to when patch area was included (Figure A7 and A8).

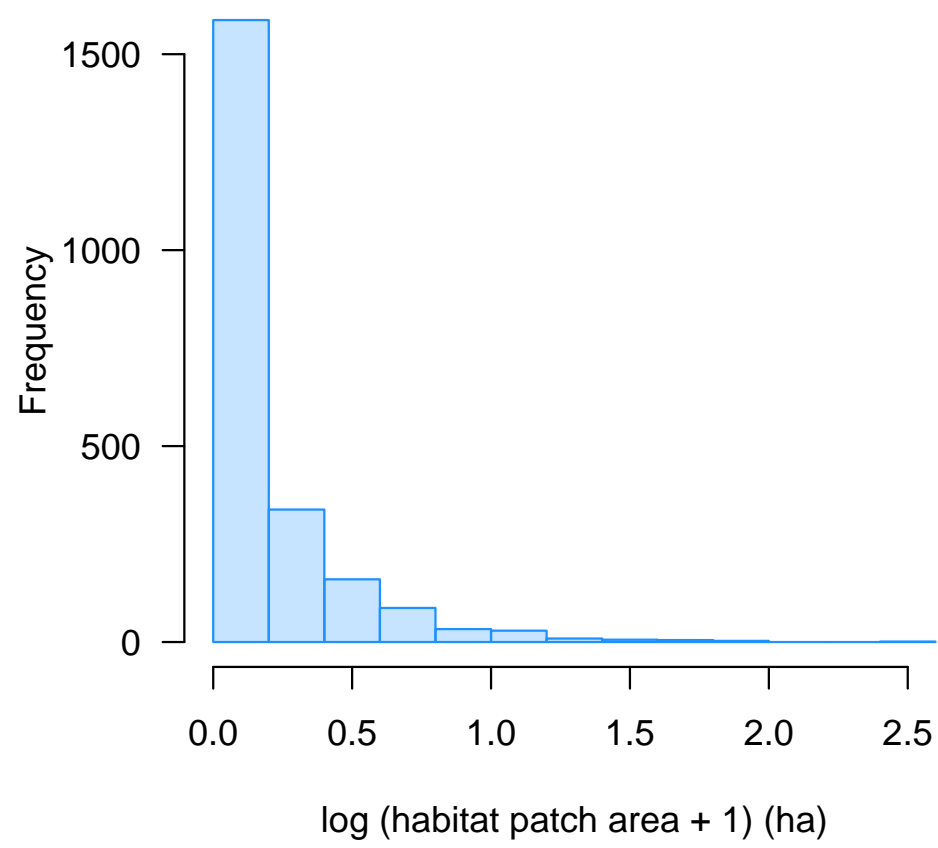

Figure A6: The distribution of habitat patch areas (log+1 transformed) in the Åland islands. 


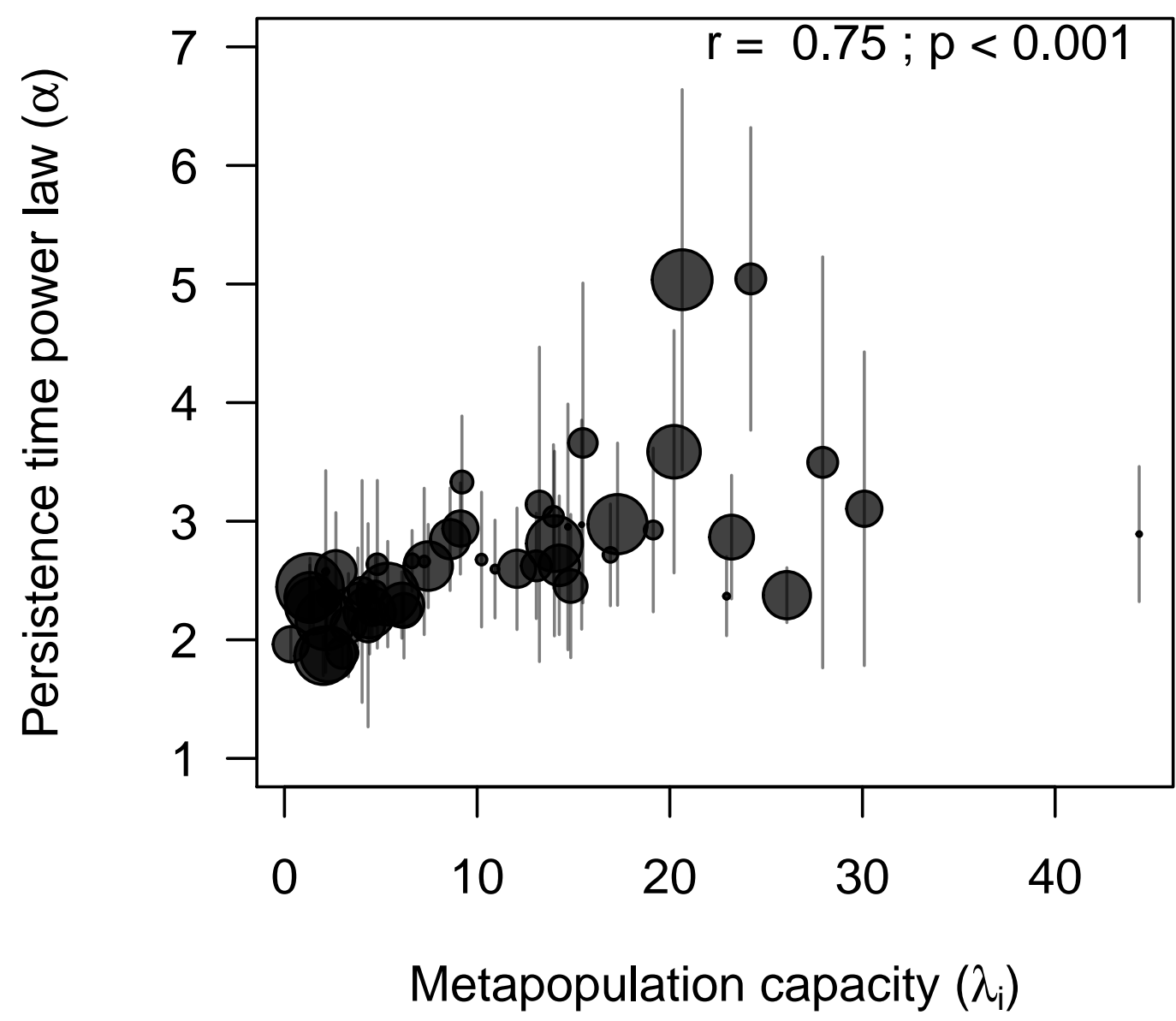

Figure A7: The relationship between metapopulation capacity $(\lambda)$ and persistence time distributions (power law fits; $\alpha$ ) for each semi-independent network (SIN). Error bars represent the estimated standard deviation in the $\alpha$ parameter, and point size is proportional to the $p$-value of the goodness of fit test for the power law fit to the persistence time distribution. Reported statistics correspond to Spearman's rank correlation coefficient and associated $p$-value. 


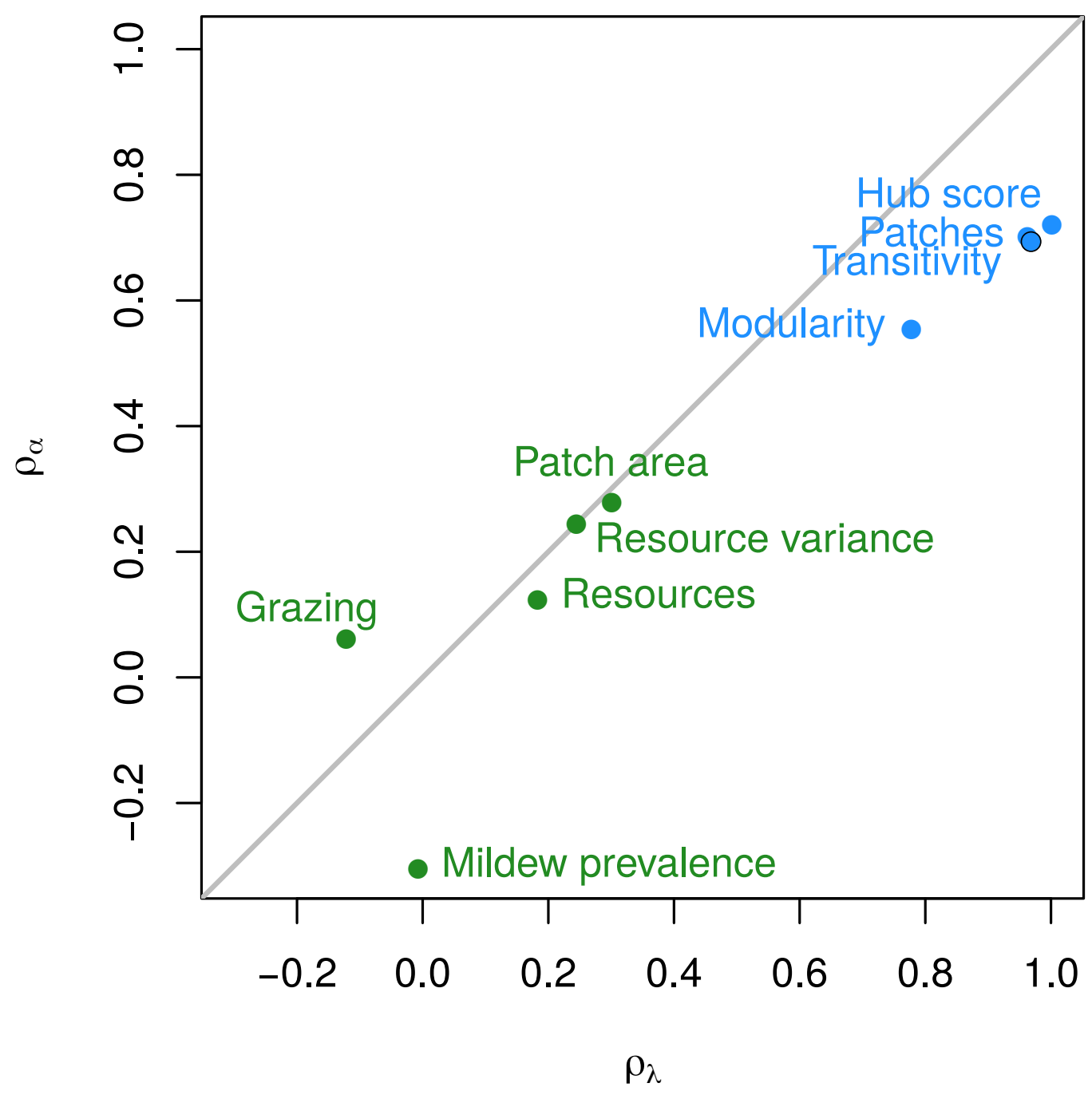

Figure A8: Spearman's correlation coefficients between network (in blue) and environmental (in green) covariates, and both measures of metapopulation persistence ((metapopulation capacity $\lambda$ and persistence time distribution fits $\alpha$ ). The grey line corresponds to an equally strong correlation with both measures of metapopulation persistence. The majority of covariates are close to this line, signaling a similar relationship between each covariate and the two persistence measures. 
Numerous relationships existed between environmental variables and metapopulation capacity, despite metapopulation capacity being based solely on the landscape matrix (M). Given this, why is metapopulation capacity often related to environmental variables? One potential reason is that collinearity among patch area and environmental variables allows environmental variation to be captured by metapopulation capacity. This can be observed in the relationship between patch area and resource availability $(r=0.30, p=0.004)$, and in the subsequent relationship between resource availability and grazing pressure $(r=-0.37, p=0.0003$; Figure A9). However, as noted in the main text, this explanation does not account for the fact that environmental correlations to metapopulation capacity (Figure A8) were maintained when patch area was removed from the estimation of the landscape matrix $(\mathbf{M})$. 


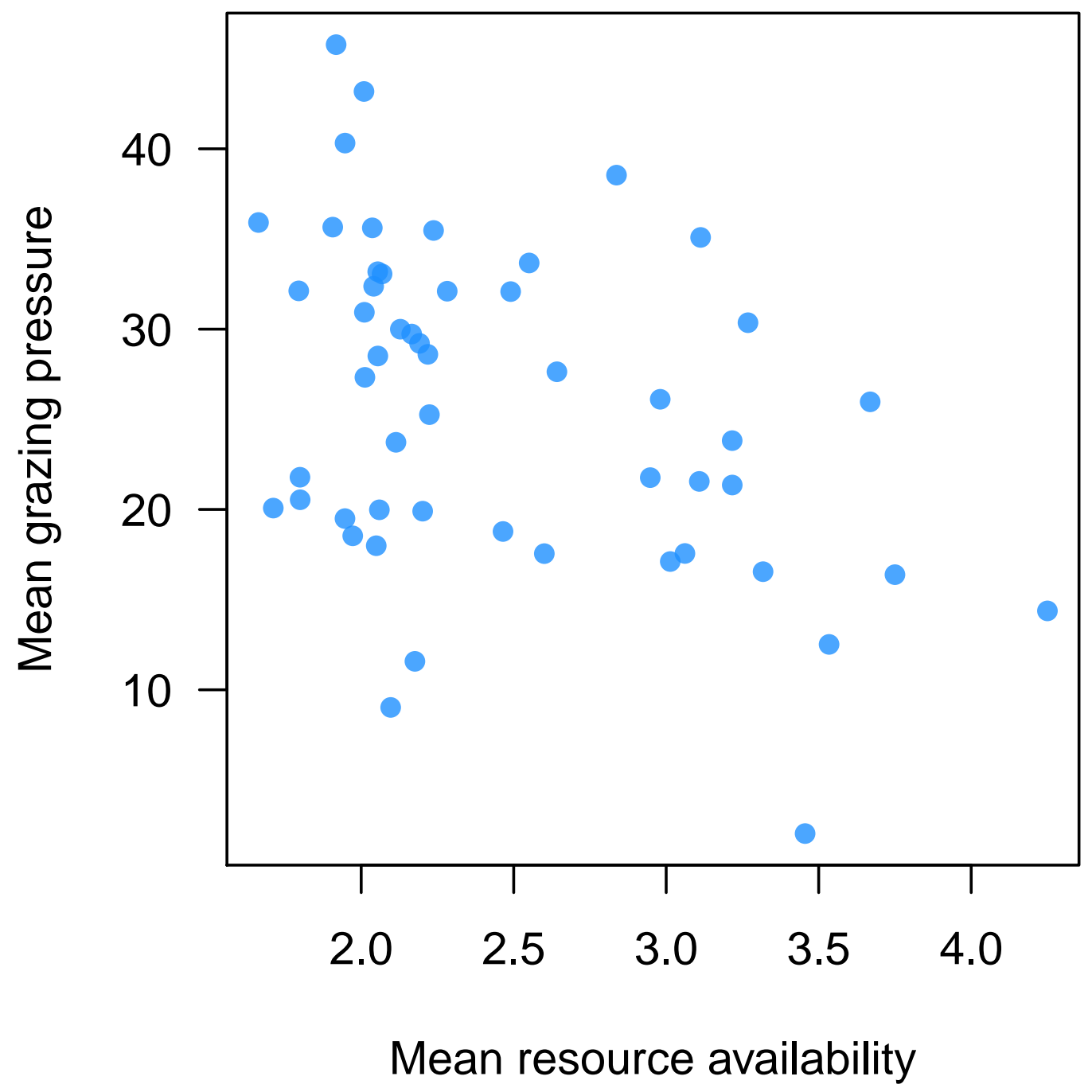

Figure A9: The relationship between mean resource availability - quantified as the mean of the sum of $V$. spicata and $P$. lanceolata abundance values across the sampling period - and mean grazing pressure over the same time period. 